\title{
Psychological Strategies for Maintaining Mental Health During the Coronavirus 2019 Pandemic
}

\author{
Mahdiyeh Sarraf-Razavi ${ }^{1}$, Seyyed Mohammad Tabatabaei ${ }^{2,3}{ }^{\text {M }}$, Mahboubeh Eslamzadeh ${ }^{1,4}$, Marjan Moradi ${ }^{4}$, \\ Zhaleh Feyzi ${ }^{5}$, Ali Talaei ${ }^{1,4}$, Mohammad Reza Fayyazi Bordbar ${ }^{1,4}$
}

\author{
Division of Neurocognitive Sciences, Psychiatry and Behavioral Sciences Research Center, Mashhad University of Medical Sciences, \\ Mashhad, Iran \\ ${ }^{2}$ Department of Medical Informatics, Faculty of Medicine, Mashhad University of Medical Sciences, Mashhad, Iran \\ ${ }^{3}$ Clinical Research Development Unit, Imam Reza Hospital, Mashhad University of Medical Sciences, Mashhad, Iran \\ ${ }^{4}$ Psychiatry and Behavioral Sciences Research Center, Mashhad University of Medical Sciences, Mashhad, Iran \\ ${ }^{5}$ Ibn-e- Sina Psychiatric Centre and Imam Reza hospital, Mashhad University of Medical Sciences, Mashhad, Iran
}

\begin{abstract}
The infection caused by the novel coronavirus (COVID-19) started from China and spread out to the whole world, and became a pandemic as the world health organization declared. COVID-19 has caused many challenges in all aspects of life, including mental health for the entire human beings. The current study has reviewed some important strategies based on individual care and social support. Maintaining healthy lifestyle and doing regular life activities such as enough sleeping, doing art, listening to music, doing yoga, and meditation could be practical for individual care. Some social support strategies may include talking to trusty people or counselors to share emotions, keeping in touch with family, friends, and colleagues, supporting people via calls and texts warmly, and taking care of the elderly, children, and especially medical workers.
\end{abstract}

[GMJ.2021;10:e2008] DOI:10.31661/gmj.v10i0.2008

Keywords: Coronavirus; Stress; Depression; Mental Health

\section{Introduction}

A ccording to the world health organization (WHO) report, the coronavirus disease 2019 (COVID-19) outbreak was recognized as an international concern for public health on 30 January 2020 when infected cases were identified in all 34 regions of China, and the disease infected more people than the severe acute respiratory syndrome (SARS) of 2003. Apparently, on late December 2019, it has started in a seafood wholesale market in the city of Wuhan in Hubei Province, then it spread rapidly within and beyond Wuhan and

\section{GMJ}

Copyright $(\mathcal{C}$ 2021, Galen Medical Journal. This is an open-access article distributed under the terms of the Creative Commons Attribution 4.0 Internation License (http://creativecommons.org/licenses/by/4.0/) Email:info@gmj.ir across the world. Regardless of exposure, experiencing fear and anxiety about getting sick or even dying, feeling helplessness, or blaming patients are potential triggers of mental breakdown [1]. Mental disorders include depression, anxiety, panic attacks, somatic symptoms and posttraumatic stress disorder, delirium, psychosis and even suicidality, worry, ruminations, despair, craving to use addictive substances, feeling guilty, insomnia, and sleep disturbances that have caused a notable number of psychiatric deaths [1-5]. Therefore, it became more important for health systems to do their best to keep their people mentally

\footnotetext{
Correspondence to:

Seyyed Mohammad Tabatabaei, Ibne Sina Street, Imam Reza Hospital, Central Library Department, Clinical Research Development Unit

Phone: Telephone Number: +989153082261

Email Address: moh.taba@gmail.com
} 
healthy. The present study investigated literatures about this matter, and tried to discuss important strategies based on individual care and social support in order to help to prevent depression and reduce stress during this pandemic for a better lifestyle during a situation like this.

\section{Search Strategy}

In order to perform this review study, searches were performed on the Google Scholar, Web of Science, Scopus, PubMed, and Embase databases for articles published from the beginning of this pandemic up to 20 September 2020, using keywords including "novel coronavirus 2019," "Stress," "depression," and "mental health maintaining." Only documents published in English containing practical strategies for maintaining mental health and reducing depression and stress during this criticizing situation were included to review. The initial search returned 63 articles, which were screened according to the aim of the current research. We selected articles mentioning strategies for a better lifestyle and keeping mental health during quarantine and this crisis. There were around 23 literatures about this issue listed in the references section.

\section{Results}

\section{Most Important Strategies Based on Own Self-Protection}

Psychological capital focuses on self-efficacy, optimism, hope, and resilience that can lead to more power and motivations, characterized by believing in being capable of doing (a "cando" spirit) and determined to do it (a "willdo" attitude) [1,6]. We can compassionately understand our emotional and psychological responses under stress and avoid emotional reactivity [2]. Being calm, taking a deep breath and using breath-centered practices may help one to relax his/her body [2,6]. Moreover, the quarantine period is a good time for yoga and meditation [7]. Coping skills, including doing daily living activities, participating in pleasurable activities, doing art, listening to music, and maintaining pride, are suggested to be considered by whoever is in quarantine [7]. It is recommended that everybody try to have a healthy diet, sleep, exercise properly, and also stay active in order to maintain a healthy lifestyle. All should take care of their mental health and try not to use tobacco, alcohol, and other drugs because of the negative emotions they cause [8-11]. The media have highlighted COVID-19 as a unique threat causing more panic, stress, and hysteria. Social media exposure, which may mistakenly spread inaccurate information and cause more fear and anxiety, should be limited to only reliable information [7]. All should be warned and concerned about suggested effects that can easily cause illness [12] and appearing symptoms at the onset of COVID-19.

\section{Most Important Strategies Based on Relation- ship and Social Support}

Real-life experiences such as team working for solving a problem, being involved in voluntary or charity activities, and providing social support for helping whoever needs may develop and strength psychological capital [1, 6]. Actually, feeling sad, stressed, confused, scared or even angry is quite normal while facing such a crisis. Talking to trustful people or even a counselor can be smoothing [8], which would permit to express negative emotions [13]. Everyone should seek out people who would respect their values and beliefs, and the spiritual practices that are uniquely meaningful to them. All should try to seek spiritual care, find trustful people around to share their spiritual, moral, and religious struggles as much as possible and offer care to the others who seek for [2]. It is strongly recommended to keep in touch with the family, friends, and colleagues in order to offer compassion and support via phone calls, texts, video chats, e-mail, or other messenger apps and provide appropriate assistance while avoiding to hostile or judge the ones who got infected with SARS-COV-2 and are in isolation $[7,14]$. Useful information must be shared with friends and family [9]. Positive and motivational stories about local people who have experienced the disease and have recovered or supported a loved one to get recovered should be highlighted and expressed. The efforts and sacrifices made by caregivers and healthcare workers who play an essential role in saving lives and keeping people safe should be remembered and appre- 
ciated [7]. A study conducted in China showed that living in urban areas, living with parents, and having a steady family income were protective factors for college students while getting anxious during the COVID-19 outbreak. The stressors relating to COVID-19, including economic influences, effects on daily-life, and academic delays, were positively associated with the level of anxiety symptoms in college students from China during the epidemic, while social support was negatively correlated with it. It means that the students require attention, help, and support provided by their community, families, and colleges [15].

\section{Elderly People and Children Require Special Care}

This can make the situation more difficult, which is already challenging for the elderly, especially for those dealing with depression and other mental disorders. Isolation of older adults, particularly those with cognitive decline or dementia, may cause more anxiety, anger, stress, agitation, and withdrawal during the quarantine period and even the outbreak. These people need to be more emotionally supported by informal support networks (families) and healthcare professionals [16]. Self-isolation would disproportionately affect the elderly who can only have social contacts at places outside their house, such as daycare venues, community centers, and worshipping places. Not having a family and/or close friend along with being alone, isolated, and secluded may increase the risk. Although there might be difficulties due to access and literacy of using novel technologies, they could be useful in providing social support networks and a sense of belonging. Interventions could be as simple as just remotely contacting others, close family and friends, voluntary organizations, and healthcare professionals during the isolation [17]. Also, virtual reality technology can improve physical and mental healthcare [18, 19]. Children may seek to be more attached and demanding on parents during the quarantine. COVID-19 should be explained to and discussed with them honestly and appropriately. Parents should help their children freely express their disturbing feelings, such as fear and sadness, which may make them feel relieved. Because every one of them may have a different way to express those emotions, it can be facilitated by engaging them to do a creative activity such as playing or drawing [16]. Online psychological consultation is necessary for people of all age groups who cannot manage their stress and anxiety during this situation. Several 24-hour psychological assistance hotlines, free of charge, available for the general public, and providing channels for citizens to relieve their negative emotions online with a psychologist are implemented in many countries. Psychological hotlines and online consultations have been proven to help community members maintaining their mental health during this outbreak $[3,14,20]$. Mental wellness and psychological intervention initiation can be promoted through face-to-face therapy as online or even offline psychoeducation about the outbreak [1]. For parents, mindfulness-based stress reduction and other psychological techniques can be adopted to relieve their anxiety and panic. This may help them get their optimistic confidence built up to overcome the disease [21]. Moreover, cognitive-behavioral therapies must be available online to defeat the feeling of loneliness and improve mental health [17]. Appropriate measures, such as providing psychological counseling services, should be defined and implemented by the government and healthcare authorities for medical staff to prevent, alleviate, and treat increased anxiety among them during the COVID-19 epidemic [14].

\section{Paying Attention to Medical Workers}

Mental health problems in medical staff, besides negatively affecting their ability to focus, understand, and make a decision, which is not good while fighting against COVID-19, could also have a long-term impact on their overall wellbeing. Thus, protecting their mental health is very important to control the epidemic and their own health [5]. Flexible schedules arrangement for medical staff, especially those affected or have an affected family member, are recommended [4, 16, 22-24].

\section{Being Respectful, Polite and Empathetic About Patient and COVID-19 Carrier People Everyone should notice that suspected and confirmed cases, and those visiting them are probably stressed or afraid. While encounter-}


ing these people, it is very important to listen to their questions and concerns very carefully while using the local language and speaking slowly. They should be answered with correct information about COVID-19. Indeed, there might be no answer to some questions since a lot is still unknown about COVID-19 [8]. Patients should be encouraged to use safe communication channels, such as smartphone communication with their families, to decrease the negative effects of social isolation and loneliness [25].

\section{Discussion}

The current study aims to discuss some psychological strategies for maintaining mental health during the COVID-19 pandemic status. The mentioned strategies would help everyone keep calm, be happy, and avoid fears and stress during this crisis. According to conducted studies on this matter, stress can cause a disturbance in the balance of hormones and cytokines and consequently weaken the immune system by reducing immune system protection cells [26]. Working in a dangerous hospital atmosphere, fear of getting sick, as well as fear of unknown aspects of the virus has led to persistent stress and subsequent suppression of the immune system and increased mortality among healthcare system staff, which are showing growing reports compared to other individuals in this epidemic [27, 28]. According to the authors, experiences, whom they are hospital staff, and the severity of COVID-19 symptoms are higher in people with high levels of fear and stress than other staff. Therefore, according to our observations and other results, keeping the immune system healthy can increase life quality and overcome this crisis.

\section{Conclusion}

Based on the available evidences, maintaining a healthy lifestyle and doing regular life activities can be practical for individual care. Some social support strategies, including talking to trusty people or a counselor and sharing emotions, keeping relationships with the family, friends, and colleagues, supporting via types of calls and texts; and taking care of the elderly, children, and especially medical workers, can help maintain mental health during the pandemic.

\section{Acknowledgment}

We would like to thank the Clinical Research Development Unit, Imam Reza Hospital, Mashhad University of Medical Sciences for their support and collaboration in conducting this study.

\section{Conflict of Interest}

The authors declare no conflict of interests.

\section{References}

1. Ho CS, Chee CY, Ho RC. Mental Health Strategies to Combat the Psychological Impact of COVID-19 Beyond Paranoia and Panic. Ann Acad Med Singap. 2020; 16;49(3):155-160.

2. Doehring C. Coping with moral struggles arising from coronavirus stress: Spiritual self-care for chaplains and religious leaders. Iliff School of Theology.(https:// s3.amazonaws.com/iliff-edu/wp-content/ uploads/2020/03/19082022/coronavirusstress.pdf).

3. Zhou X. Psychological crisis interventions in Sichuan Province during the 2019 novel coronavirus outbreak. Psychiatry Res. 2020;
26;286:112895.

4. Lai J, Ma S, Wang Y, et al. Factors Associated With Mental Health Outcomes Among Health Care Workers Exposed to Coronavirus Disease 2019. JAMA Netw Open. 2020;3(3):e203976.

5. Kang L, Li Y, Hu S, Chen M, Yang C, Yang $\mathrm{BX}$, et al. The mental health of medical workers in Wuhan, China dealing with the 2019 novel coronavirus. Lancet Psychiatry. 2020; 7(3):e14.

6. CHAN D. "The $5 \mathrm{Cs}$ of beating the coronavirus outbreak" (2020). Research Collection School of Social Sciences. Paper 3168. (https://ink.library.smu.edu.sg/soss 
research/3168).

7. Jiloha RC. COVID-19 and Mental Health. Epidemiology International. 2020;5(1): 7-9.

8. World Health Organization. Regional Office for the Western Pacific. (2020)? The COVID-19 risk communication package for healthcare facilities. Manila: WHO Regional Office for the Western Pacific. https://apps. who.int/iris/handle/10665/331140. License: CC BY-NC-SA 3.0 IGO.

9. Wiederhold K.W. Using Social Media to Our Advantage: Alleviating Anxiety During a Pandemic. Cyberpsychology, Behavior, and Social Networking. 2020; 23(4); 197-198.

10. Zhu W. Should, and how can, exercise be done during a coronavirus outbreak? An interview with Dr. Jeffrey A. Woods. J Sport Health Sci. 2020;9(2):105-107.

11. Xiao H, Zhang Y, Kong D, Li S, Yang N. Social Capital and Sleep Quality in Individuals Who Self-Isolated for 14 Days During the Coronavirus Disease 2019 (COVID-19) Outbreak in January 2020 in China. Med Sci Monit. 2020; 20;26:e923921.

12. Lorber W, Mazzoni G, Kirsch I. Illness by suggestion: expectancy, modeling, and gender in the production of psychosomatic symptoms. Ann Behav Med. 2007;33(1):1126.

13. Park SC, Park YC. Mental Health Care Measures in Response to the 2019 Novel Coronavirus Outbreak in Korea. Psychiatry Investig. 2020;17(2):85-86.

14. Zhang J, Wu W, Zhao X, and Zhang W. Recommended psychological crisis intervention response to the 2019 novel coronavirus pneumoniaoutbreak in China: a model of West China Hospital. Precision Clinical Medicine. 2020;3(1): 3-8.

15. Cao W, Fang Z, Hou G, Han M, Xu X, Dong $\mathrm{J}$, et al. The psychological impact of the COVID-19 epidemic on college students in China. Psychiatry Res. 2020; 287:112934.

16. Liu C, Yang Y-Z, Zhang X M, Xu X, Dou Q-L, Zhang W W. The prevalence and influencing factors for anxiety in medical workers fighting COVID-19 in China: A cross-sectional survey. Epidemiol Infect. 2020;148:e98.

17. Armitage R, Nellums LB. COVID-19 and the consequences of isolating the elderly. Lancet Public Health. 2020; 5(5):e256.

18. Sarraf-Razavi M, Tabatabaei M, Talaei A, Fayyazi Bordbar MR. Application of Virtual Reality for Helping People with Psychiatric Disorders: A Mini Review. RMES. 2019;
000684. 8(2):867-869.

19. Tabatabaei M, and Talaei A. Virtual Reality as a Friend of the Elderly: A Mini-Review. Biomed J Sci \& Tech Res. 22(2)-2019.

20. Zhu Y, Chen L, Ji H, Xi M, Fang Y, Li Y. The Risk and Prevention of Novel Coronavirus Pneumonia Infections Among Inpatients in Psychiatric Hospitals. Neurosci Bull. 2020;36(3):299-302.

21. Jin YH, Cai L, Cheng ZS, Cheng H, Deng T, Fan YP, and et.al. A rapid advice guideline for the diagnosis and treatment of 2019 novel coronavirus (2019-nCoV) infected pneumonia (standard version). Mil Med Res. 2020;6;7(1):4.

22. Ying Y, Ruan L, Kong F, Zhu B, Ji Y, Lou Z. Mental health status among family members of health care workers in Ningbo, China, during the coronavirus disease 2019 (COVID-19) outbreak: a cross-sectional study. BMC psychiatry. 2020;20(1):1-0.

23. Cai H, Tu B, Ma J, Chen L, Fu L, Jiang Y, and et al. Psychological Impact and Coping Strategies of Frontline Medical Staff in Hunan Between January and March 2020 During the Outbreak of Coronavirus Disease 2019 (COVID 19) in Hubei, China. Med Sci Monit. 2020;15;26:e924171.

24. Xiao H, Zhang Y, Kong D, Li S, Yang N. The Effects of Social Support on Sleep Quality of Medical Staff treating Patients with Coronavirus Disease 2019 (COVID-19) in January and February 2020 in China. Med Sci Monit. 2020; 5;26:e923549.

25. Xiang YT, Yang Y, Li W, Zhang L, Zhang Q, Cheung T, et al. Timely mental health care for the 2019 novel coronavirus outbreak is urgently needed. Lancet Psychiatry. 2020;7(3):228-229.

26. Lai J, Ma S, Wang Y, Cai Z, Hu J, Wei N, and et al. Factors associated with mental health outcomes among health care workers exposed to coronavirus disease 2019.JAMA Netw Open. 2020; 3 (3), e203976.

27. Ehrlich H, Kenney M, Elkbuli A. Protecting our healthcare workers during the COVID-19 pandemic. (2020). In Press, Corrected Proof. Available online 17 April.

28. Mao L, Jin H, Wang M, Hu Y, Chen S, He Q, et al. Neurologic Manifestations of Hospitalized Patients With Coronavirus Disease 2019 in Wuhan, China. JAMA Neurol. 2020;1;77(6):683-690. 